\title{
Polar Oxide Interface Characterization by Differential Phase Contrast STEM
}

\author{
N. Shibata, ${ }^{1,2}$ S.D. Findlay ${ }^{3}$ and Y. Ikuhara ${ }^{1,4}$ \\ ${ }^{1}$ Institute of Engineering Innovation, The University of Tokyo, Tokyo 113-8656 Japan \\ ${ }^{2}$ PRESTO, Japan Science and Technology Agency, Saitama 332-0012, Japan \\ ${ }^{3}$ School of Physics, Monash University, Victoria 3800, Australia \\ ${ }^{4}$ Nanostructures Research Laboratory, Japan Fine Ceramic Center, Nagoya, 456-8587, Japan
}

Understanding the atomic-scale structure of surfaces and interfaces is essential to control the functional properties of many materials and devices. Recent advances in aberration-corrected scanning transmission electron microscopy (STEM) have made possible the direct characterization of localized atomic structure in ceramic materials, especially grain boundaries and heterointerfaces. In STEM, a finely focused electron probe is scanned across the specimen and the transmitted and/or scattered electrons from a localized volume of the material are detected by the post-specimen detector(s) as a function of raster position. By controlling the detector geometry, we gain flexibility in determining the contrast characteristics of the STEM images and the formation mechanisms involved. Thus, it may be possible to obtain further useful information by exploring new detector geometries in atomic-resolution STEM. Recently, we have proposed new imaging possibilities by controlling detector geometries [1-5]: annular bright-field (ABF) imaging and atomic-resolution differential phase contrast (DPC) imaging. ABF-STEM imaging enables us to directly image light element atomic columns at interfaces of ceramics materials. DPC-STEM imaging can be used to detect local electric fields, even at atomic dimensions.

In this presentation, we review our recent research on the development of atomic-resolution STEM imaging techniques and their application to polar interface characterization. Figure 1 shows a lowmagnification DPC STEM image of 90 degree domain structure in a ferroelectric $\mathrm{BaTiO}_{3}$ crystal [4]. We can clearly see contrast differences between the domains, which reflect the difference in the electric field directions and thus the polarization directions shown by the red arrows. The observed electric fields are a consequence of the imperfect screening of depolarization fields inside each ferroelectric domain, and thus contain important information about the material structure and properties. Figure 2 shows the DPC STEM image of a 180 degree domain wall in ferroelectric $\mathrm{LiTaO}_{3}$ [6]. It is clear that the DPC image obtained parallel to the polarization axis in (a) shows contrast changes between the upper and lower domains, while the DPC image obtained perpendicular to the polarization axis in (b) shows almost no contrast. These contrast changes sensitively reflect the electric field distribution inside domains in ferroelectric LiTaO3 and clearly shows that this wall is of head-to-head type. We will also consider the detailed electric field distribution across the domain wall. Finally, we will discuss how the DPC STEM imaging techniques can help us further understand the structure and functionality of polar oxide interfaces.

\section{References}

[1] S.D. Findlay et al., Appl. Phys. Lett. 95, 191913 (2009).

[2] S. D. Findlay et al., Ultramicroscopy 110, 903 (2010).

[3] N. Shibata et al., J. Electron Microscopy 59, 473 (2010).

[4] N. Shibata et al., Nature Physics, 8, 611-615 (2012).

[5] S.D. Findlay et al., Ultramicroscopy, 136, 31 (2014).

[6] N. Shibata et al., submitted. 
[7] We thank T. Matsumoto (U. Tokyo) for useful discussion and Y. Kohno, H. Sawada and Y. Kondo (JEOL Ltd.) for their collaboration in the detector development. This work was supported by the PRESTO, JST and the JSPS KAKENHI Grant number 23686093. A part of this work was conducted in Research Hub for Advanced Nano Characterization, The University of Tokyo, under the support of "Nanotechnology Platform" (project No.12024046) by MEXT, Japan. This research was supported under the Discovery Projects funding scheme of the Australian Research Council (Project No. DP110101570).
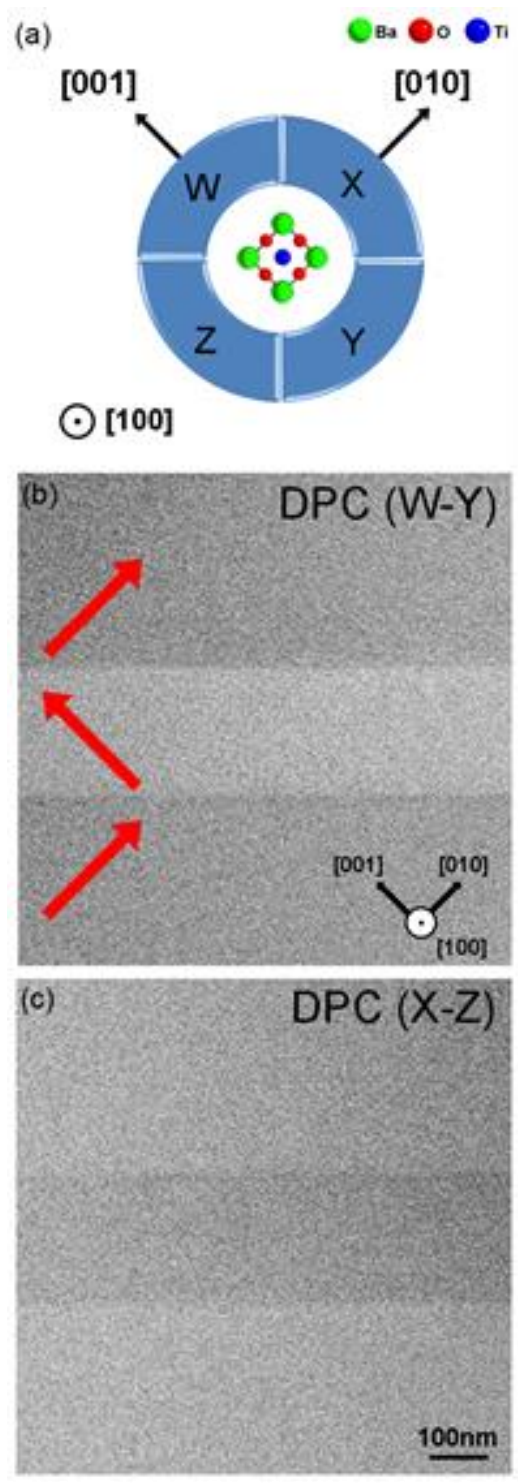

Fig. 1. (a) Schematic illustration showing the relationship between the crystallographic orientation of $\mathrm{BaTiO} 3$ and the four detector segments. (b)(c) DPC STEM images of 90 degree domain structure. (a)
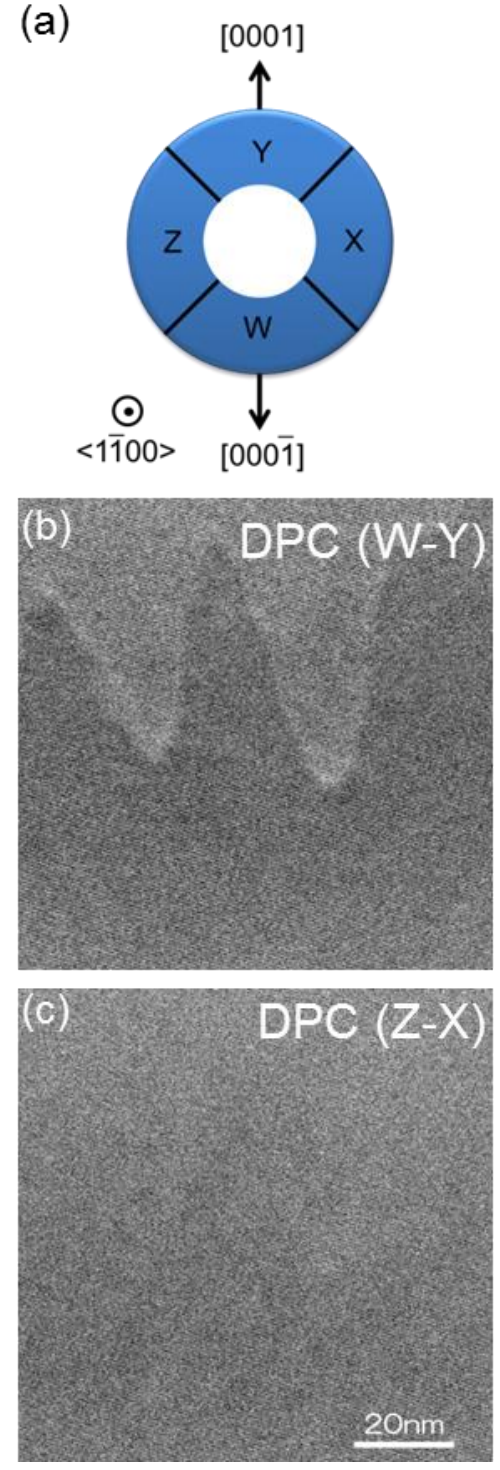

Fig. 2. (a) Schematic illustration showing the relationship between the crystallographic orientation of $\mathrm{LiTaO} 3$ and the four detector segments. (b)(c) DPC STEM images of a 180 degree domain wall. 